\title{
Succinyl-CoA-based energy metabolism dysfunctions in chronic heart failure
}

\section{Shingo Takada ( $\nabla$ s-takada@hotmail.co.jp )}

Hokkaido University Graduate School of Medicine https://orcid.org/0000-0002-7781-9482

\section{Satoshi Maekawa}

Department of Cardiovascular Medicine, Faculty of Medicine and Graduate School of Medicine, Hokkaido University

\section{Takaaki Furihata}

Department of Cardiovascular Medicine, Faculty of Medicine and Graduate School of Medicine, Hokkaido University

\section{Naoya Kakutani}

Department of Cardiovascular Medicine, Faculty of Medicine and Graduate School of Medicine, Hokkaido University

\section{Daiki Setoyama}

Department of Clinical Chemistry and Laboratory Medicine, Graduate School of Medical Sciences, Kyushu University

\section{Koji Ueda}

Japanese Foundation For Cancer Research https://orcid.org/0000-0001-9066-4959

\section{Hideo Nambu}

Department of Cardiovascular Medicine, Faculty of Medicine and Graduate School of Medicine, Hokkaido University

\section{Hikaru Hagiwara}

Department of Cardiovascular Medicine, Faculty of Medicine and Graduate School of Medicine, Hokkaido University

\section{Haruka Handa}

Hokkaido University https://orcid.org/0000-0002-8557-7479

\section{Yoshizuki Fumoto}

Department of Molecular Biology, Faculty of Medicine and Graduate School of Medicine, Hokkaido University

\section{Soichiro Hata}

Department of Molecular Biology, Faculty of Medicine and Graduate School of Medicine, Hokkaido University

\section{Arata Fukushima}

Hokkaido University Graduate School of Medicine

\section{Takashi Yokota}


Hokkaido University Graduate School of Medicine https://orcid.org/0000-0001-5470-6648

\section{Dongchon Kang}

Graduate School of Medical Sciences, Kyushu University

\section{Shintaro Kinugawa}

Department of Cardiovascular Medicine, Faculty of Medicine and Graduate School of Medicine, Hokkaido University

\section{Hisataka Sabe}

Hokkaido University Graduate School of Medicine

\section{Article}

\section{Keywords:}

Posted Date: December 7th, 2021

DOI: https://doi.org/10.21203/rs.3.rs-838227/v1

License: (c) (1) This work is licensed under a Creative Commons Attribution 4.0 International License. Read Full License 
1 Succinyl-CoA-based energy metabolism dysfunctions in chronic heart

2 failure $(74<75$ characters/spaces $)$

3

4 Shingo Takada ${ }^{1,2,3, \dagger^{*} \bowtie}$, Satoshi Maekawa ${ }^{1 \dagger}$, Takaaki Furihata ${ }^{1}$, Naoya Kakutani ${ }^{1}$,

5 Daiki Setoyama $^{4}$, Koji Ueda ${ }^{5}$, Hideo Nambu ${ }^{1}$, Hikaru Hagiwara ${ }^{1}$, Haruka Handa ${ }^{2}$,

6 Yoshizuki Fumoto ${ }^{2}$, Soichiro Hata $^{2}$, Arata Fukushima ${ }^{1}$, Takashi Yokota ${ }^{1}$, Dongchon

7 Kang $^{4,6}$, Shintaro Kinugawa ${ }^{1,7^{*} \bowtie}$, and Hisataka Sabe ${ }^{2^{*} \bowtie}$

8

$9 \quad{ }^{1}$ Department of Cardiovascular Medicine, ${ }^{2}$ Department of Molecular Biology, Hokkaido

10 University Graduate School of Medicine, Sapporo, Japan; ${ }^{3}$ Department of Lifelong Sport,

11 School of Sports Education, Hokusho University, Ebetsu, Japan; ${ }^{4}$ Department of Clinical

12 Chemistry and Laboratory Medicine; ${ }^{5}$ Cancer Precision Medicine Center, Japanese

13 Foundation for Cancer Research, Tokyo, Japan; ${ }^{6}$ Clinical Laboratories, Kyushu

14 University Hospital, Fukuoka, Japan; ${ }^{7}$ Department of Cardiovascular Medicine, Graduate

15 School of Medical Sciences, Kyushu University, Fukuoka, Japan

16

$17 \dagger$ These authors contributed equally to this work.

18 - 18 -mail:

19 *s-takada@hokusho-u.ac.jp (S.T.);

20 *kinugawa@cardiol.med.kyushu-u.ac.jp (S.K.);

21 *sabeh@med.hokudai.ac.jp (H.S.)

22 


\section{(Abstract)}

Heart failure (HF) is a leading cause of death and repeated hospitalizations ${ }^{1}$. HF progression generally involves mitochondrial dysfunction ${ }^{2-4}$. However, how mitochondria react to chronic HF remains unclear. Here, we show the molecular basis of mitochondrial dysfunction in chronic $\mathrm{HF}$, which is characterized by altered succinyl-CoA metabolism. In myocardial mitochondria of coronary ligated mice, heme synthesis and ketolysis, and enzymes using succinyl-CoA in these events were upregulated, and enzymes synthesizing succinyl-CoA at the tricarboxylic acid (TCA) cycle were also increased. Intriguingly, the ADP-specific, but not the GDP-specific, subunit of succinyl-CoA synthetase, which uses succinyl-CoA in the TCA cycle, was decreased. Myocardial succinyl-CoA levels were significantly reduced in chronic HF, impairing mitochondrial oxidative phosphorylation (OXPHOS). Consequently, the administration of 5-aminolevulinic acid $(\mathrm{ALA})^{5}$, an intermediate in the pathway from succinyl-CoA to heme synthesis, prevented HF progression in mice. Previous reports also support the presence of succinyl-CoA metabolism abnormalities in HF patients $^{6,7}$. Our results indicated that changes in succinyl-CoA usage in various energy production systems in myocardial mitochondria is characteristic to chronic HF, and that although similar alterations occur in healthy conditions, such as during strenuous exercise, they may often occur irreversibly in HF. Moreover, nutritional interventions compensating the metabolic changes are likely to provide effective methods to treat HF. $\quad(207<$ approx. 200 words $)$ 
(text)

Mitochondrial dysfunction is frequently involved in the development of HF, and metabolic dysfunction, including that of the TCA cycle, a core metabolic pathway for producing ATP, is known as the main cause ${ }^{2-4}$. Recent studies have shown that the selective accumulation of succinate, an intermediate of the TCA cycle, during acute ischemia in the mouse heart is a major cause of reperfusion injury ${ }^{8-10}$. On the other hand, how the TCA cycle and its associated energy-producing metabolic pathways respond to chronic HF remains largely unclear. We here addressed this question with the aim of understanding the metabolic basis of mitochondrial dysfunction in chronic HF. We used a mouse model of HF, in which myocardial infarction (MI) was induced by permanent left anterior descending coronary artery ligation ${ }^{11,12}$. Mice with permanent coronary artery ligation (hereafter referred to as MI mice) generally start to show HF symptoms 7 days after the ligation ${ }^{11,12}$, which we also confirmed in this study (Extended Data Fig. 1). Sham-operated mice were used as a control ${ }^{11,12}$. We used these mice 28 days after surgery

59 in all the following analyses, unless otherwise described, and excluded necrotic areas of 60 MI mice from biochemical analyses. 


\section{Extended data Fig. 1}
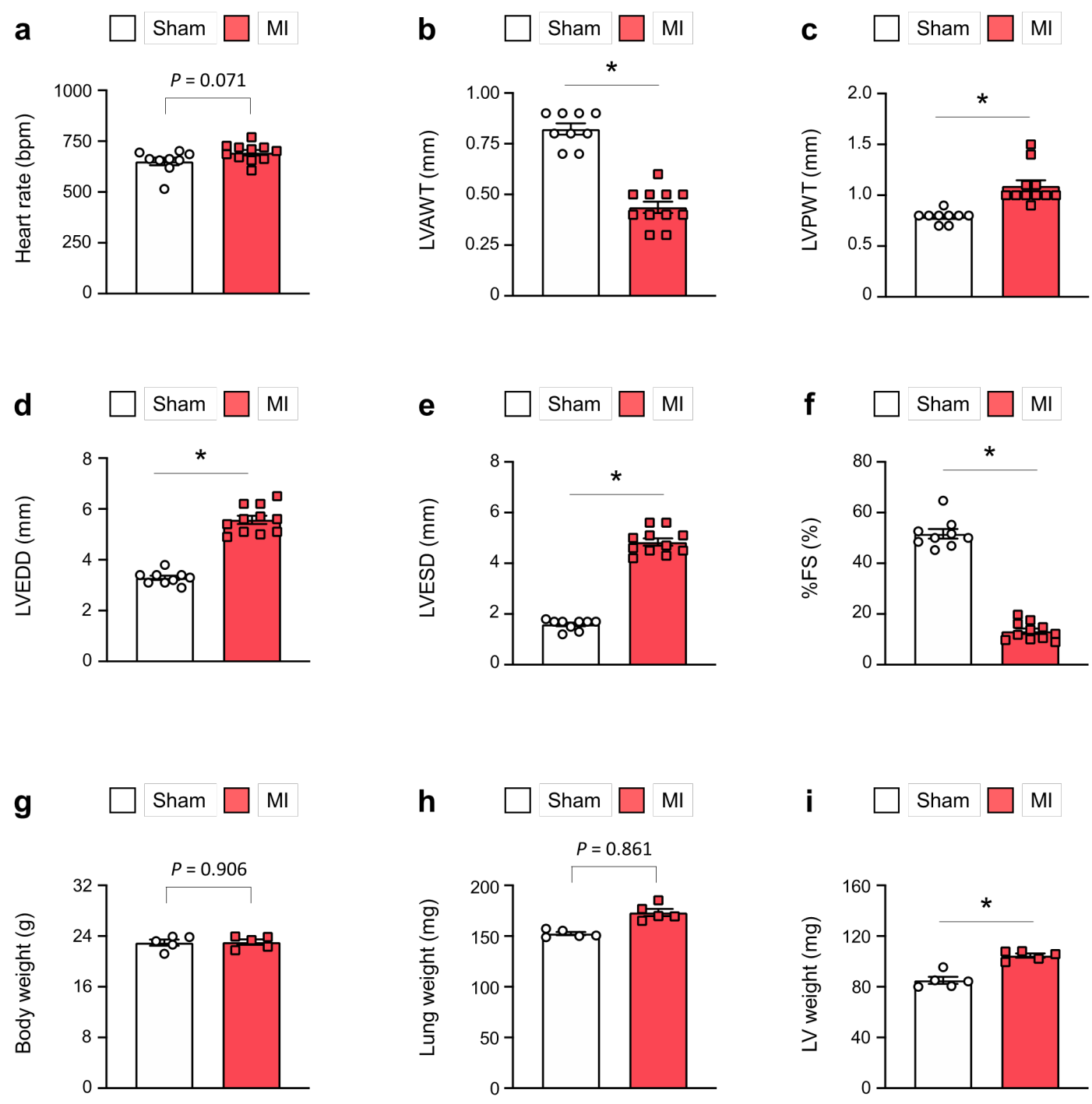

Extended Data Figure 1. Cardiac parameters of the MI mice and sham mice used in this study. MI mice and sham mice used in our experiments were randomly sampled, and

64 their cardiac parameters, namely heart rate (a), LV anterior wall thickness (LVAWT) (b),

65 LV posterior wall thickness (LVPWT) (c), LV end-diastolic diameter (LVEDD) (d), LV

66 end-systolic diameter (ESD) (e), \%FS (f), body weight (g), lung weight (h), and LV

67 weight (i). 


\section{Succinyl-CoA decreases in chronic HF (37/40 characters)}

71 To investigate the possible metabolic changes in the mouse heart during chronic HF, we 72 first analyzed the levels of TCA cycle intermediates. Liquid chromatography-mass 73 spectrometry (LC-MS)-based analysis demonstrated a significant selective reduction in 74 succinyl-CoA level in cardiac muscles of MI mice compared with those of sham mice 75 (Fig. 1). The majority of succinyl-CoA is produced by the TCA cycle ${ }^{13}$. Levels of other 76 TCA cycle intermediates, including succinate, did not show a statistically significant 77 difference between MI mice and sham mice (Fig. 1). Therefore, it is likely that metabolic 78 changes occur in the myocardial muscle of mice during chronic HF that are very different 79 from those that occur during acute ischemia ${ }^{8}$. 
Fig. 1
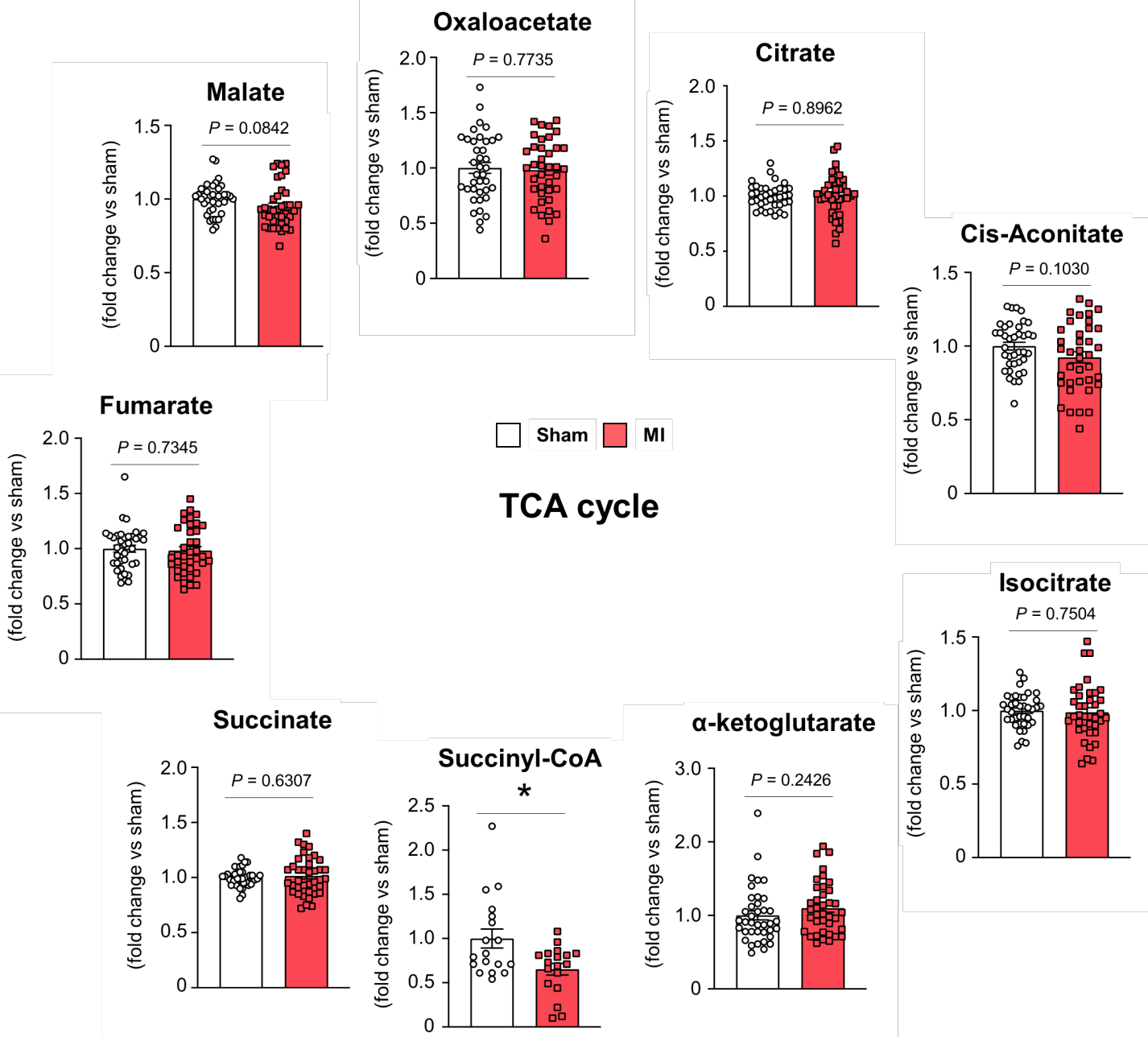

80

Fig. 1. Selective reduction of succinyl-CoA among the TCA cycle metabolites in cardiac muscle during chronic HF. Relative levels of the TCA cycle metabolites in cardiac muscles isolated from MI mice, compared with those from sham mice, 28 days after surgery. Each data point in the dot plot represents one individual mouse sample $(n=$

8538 for both groups for all metabolites, except for succinyl-CoA in which $n=18$ for both groups). Data are shown as the mean \pm s.e.m. The two-tailed Student $t$-test was performed

87 for pairwise comparisons. ${ }^{*} P<0.05$. 


\section{Enzyme levels change in chronic HF (34/40 characters)}

91 We then sought to understand the molecular bases of this reduction in succinyl-CoA level.

92

There are several other pathways that utilize succinyl-CoA in the mitochondrion, 
namely, heme synthesis and ketolysis ${ }^{13}$. Heme is essential for mitochondrial OXPHOS. Mitochondrial heme synthesis is initiated from the synthesis of ALA, which is synthesized from succinyl-CoA and glycine by the mitochondrial rate-limiting enzyme 5'-aminolevulinate synthase 1 (Alas1) (ref. ${ }^{5,14}$ ) (see Fig. 2h). In the myocardial mitochondria of MI mice, levels of Alas1 and heme were significantly increased compared with sham mice (Fig. 2i, j). On the other hand, it is well documented that the utilization of ketone bodies as an energy source is upregulated in the heart in proportion to the level of plasma ketone bodies, accompanied by the increased production of ketone bodies in the liver, in both HF patients and animal models ${ }^{15,16}$. In mitochondrial ketolysis, ketone bodies are converted into acetyl-CoA (see Fig. 2k). Consistently, $\beta$ hydroxybutyric acid ( $\beta$-OHB), a major component of ketone bodies, was significantly increased in the plasma but was significantly reduced in the cardiac muscles of MI mice compared with sham mice (Fig. 21, m). Moreover, the level of 3-oxoacid CoA-transferase 1 (Oxct1), which is the main catalyst of ketone bodies via the use of succinyl-CoA (see Fig. 2k), was significantly upregulated in the myocardial mitochondria of MI mice (Fig. 2n). Therefore, collectively, our results suggest that the myocardial mitochondria of mice undergo metabolic changes during chronic HF, in which levels of enzymes that utilize succinyl-CoA for heme synthesis and ketolysis are increased, and the level of an enzyme that utilizes succinyl-CoA in the TCA cycle is decreased. Moreover, although these changes also involve increased levels of enzymes that synthesize succinyl-CoA from $\alpha \mathrm{KG}$ and glutamate, our results suggested that these changes often result in reduced succinylCoA levels. Binding sites of hypoxia-inducible factor 1 are found in the ALAS1/Alas 1 promoter regions in both humans and mice (http://jaspar.genereg.net/), although ALAS1 mRNA is not always upregulated under hypoxia ${ }^{17}$. Increased heme levels in the failing 
137 hearts of mice have also been reported previously, but the upregulation of Alas 1 has not

138 been shown to date ${ }^{18}$. Downregulation of the forward reaction of the TCA cycle may

139 suppress production of reactive oxygen species in mitochondria under lowered oxygen

140 concentrations $^{8}$. Moreover, not only failing hearts, but some other organs, including

141 skeletal muscle under healthy conditions also utilize ketone bodies, such as during long-

142 lasting vigorous physical exercise ${ }^{19}$, although whether levels of the enzymes involved

143 also change in healthy conditions is unknown. Thus, taken together, myocardial

144 mitochondria in chronic HF may undergo a hitherto unknown mode of metabolic

145 dysfunction, although many of the events involved in this dysfunction are known

146 physiological responses of mitochondria. It should be noted, however, that many issues

147 remain unsolved, including the molecular basis of the altered enzyme levels in chronic

$148 \mathrm{HF}$, as well as whether these metabolic shifts are associated with cardiac remodeling

149 during chronic HF. 
a
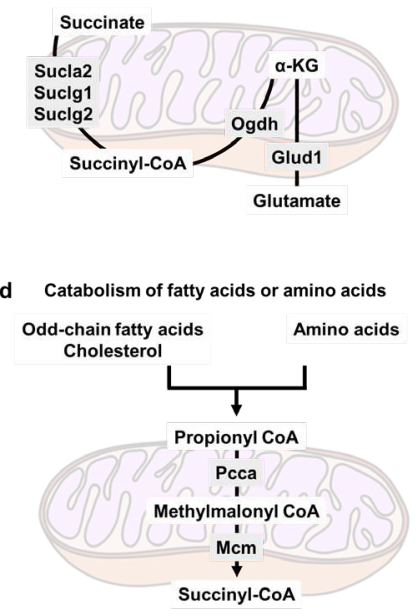

g
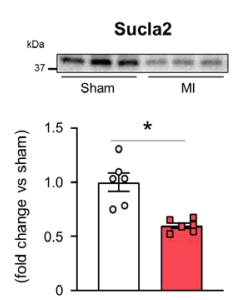

h
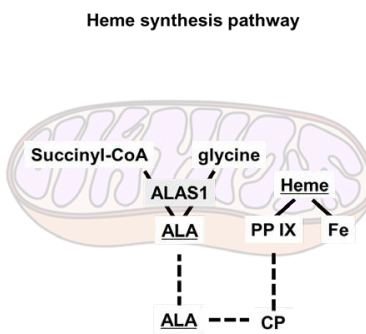

$\mathbf{k}$

Ketolytic pathway

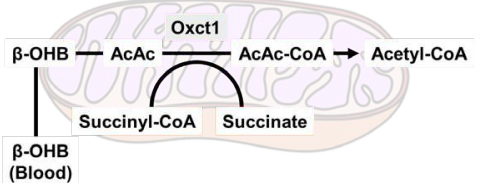

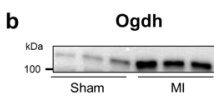
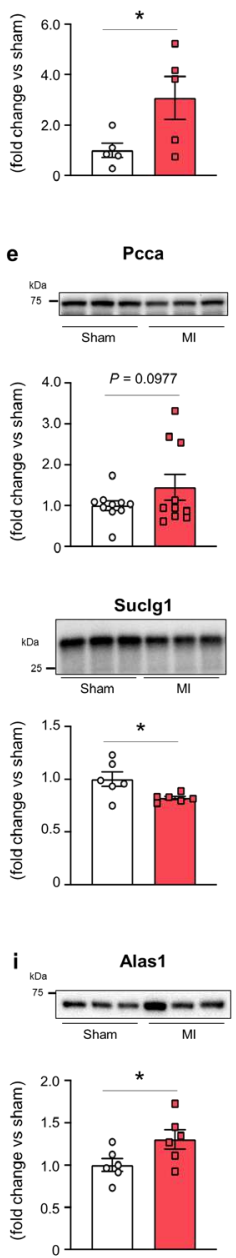

I $\quad$ OOHB in plasma

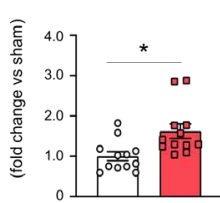

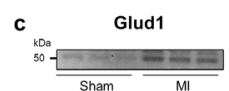
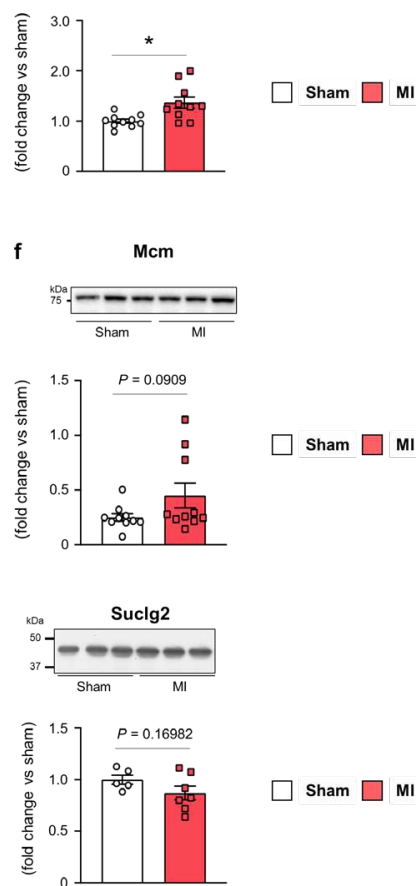

j Mitochondrial heme
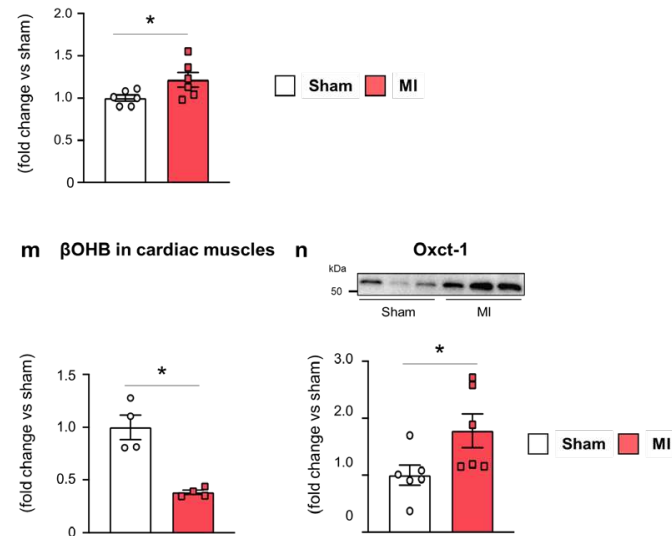

Fig. 2. Enzyme level changes in myocardial mitochondria during chronic HF promote heme synthesis and ketolysis, and downregulate the incorporation of succinyl-CoA into the TCA cycle. a-c, Succinyl-CoA metabolism in the TCA cycle (a);

154 and relative protein levels of $\operatorname{Ogdh}(\mathbf{b})(n=5)$ and Glud1 $(\mathbf{c})(n=10)$. d-f, Propionyl-

155 CoA-based succinyl-CoA synthesis in the mitochondrion (a); and relative protein levels 
of Pcca $(\mathbf{e})(n=10)$ and Mcm (f) $(n=10)$. g, Relative protein levels of the succinyl-CoA synthetase subunits Sucla2 (left, $n=6$ ), Suclg1 (middle, $n=6$ ) and Suclg2 (right, $n=6$ ). $\mathbf{h}-\mathbf{j}$, Heme synthesis pathway in the mitochondrion (h); and relative protein levels of Alas1 (i) $(n=6)$ and relative amounts of heme $(\mathbf{j})(n=6)$. CP, coproporphyrinogen-III; and PPIX, protoporphyrin IX. k-n, Ketolytic pathway in the mitochondrion (k); and relative amounts of $\beta$-OHB in plasma (l) $(n=12)$ and cardiac muscle $(\mathbf{m})(n=4)$, and relative levels of Oxct1 in mitochondria $(\mathbf{n})(n=6)$. All assays were performed using isolated myocardial mitochondria, except for I (blood plasma) and $\mathbf{m}$ (cardiac muscle). In $\mathbf{b}, \mathbf{c}, \mathbf{e}, \mathbf{f}, \mathbf{g}, \mathbf{i}$, and $\mathbf{n}$, representative results of each immunoblot blot are shown in the upper panels. Each data point in the dot plot represents one individual mouse sample. Data are shown as the mean \pm s.e.m. The two-tailed Student $t$-test was performed for pairwise comparisons. ${ }^{*} P<0.05$. Full-size CBB staining scans of the immunoblots are shown in Extended Data Fig. 5.

\section{Succinyl-CoA reduction impairs OXPHOS (38/40 characters)}

We then addressed whether a decreased succinyl-CoA level impairs mitochondrial OXPHOS capacity. We previously showed that both complex I (CI)-linked (i.e., NADHdriven $\mathrm{CI} /$ complex III [CIII]/complex IV [CIV] supercomplex) and complex II (CII)linked (i.e., FADH-driven CII/CIII/CIV chain) OXPHOS capacities are decreased in the myocardial mitochondria of MI mice ${ }^{20}$, which we hereby confirmed again (Fig. 3a). The addition of $1 \mathrm{mM}$ succinyl-CoA to isolated myocardial mitochondria of MI mice increased their CI- and CII-linked OXPHOS capacities to levels almost comparable to those of sham mice (Fig. 3a). Myocardial mitochondria isolated from non-operated mice 
180

181

182

did not show this positive response to succinyl-CoA (Fig. 3b), and even $3 \mathrm{mM}$ and 10 mM succinyl-CoA still did not increase OXPHOS activity, but rather impaired it (Fig. 3b). On the other hand, 1 to $10 \mathrm{mM}$ succinate did not show such effects (Fig. 3c). Therefore, these results indicate that the decrease in succinyl-CoA level in the cardiac muscle of mice during chronic HF is likely to contribute to the reduced CI- and CII-linked OXPHOS capacities of the failing heart. CI requires NADH for its activity, and CII is driven by succinate dehydrogenase $(\mathrm{Sdh})^{2,21}$. Thus, our results imply that the decreased level of succinyl-CoA affects some reactions of the TCA cycle that produce NADH, and also downregulates the forward reaction of Sdh. Impaired CII activity, as well as the increased level of Oxct1, which generates succinate from succinyl-CoA, of the myocardial mitochondria of MI mice would explain, at least in part, why the level of succinate was not notably changed, despite the reduced succinyl-CoA level. On the other hand, our results posed the simple question as to why the external addition of succinyl-CoA did not upregulate the OXPHOS capacity of intact mitochondria, although it upregulated this capacity in the myocardial mitochondria of MI mice. 
a

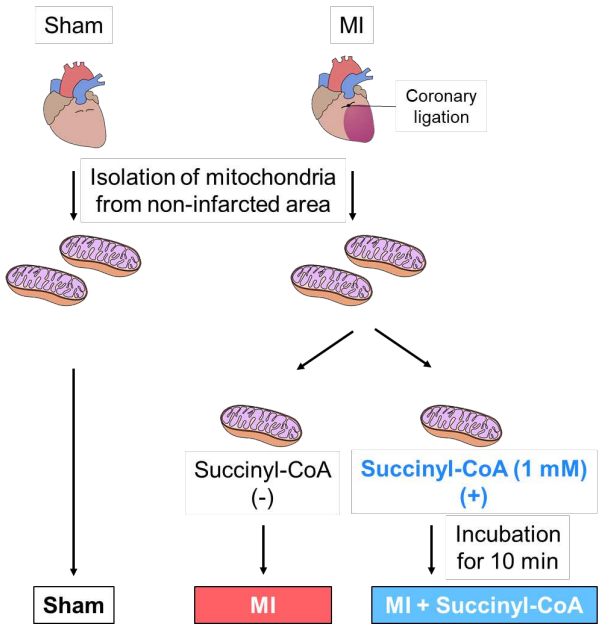

C

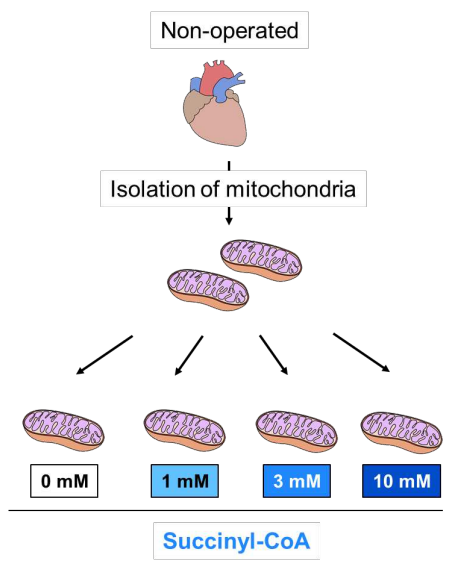

e

Non-operated

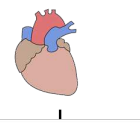

Isolation of mitochondria

$\downarrow$

एवराल
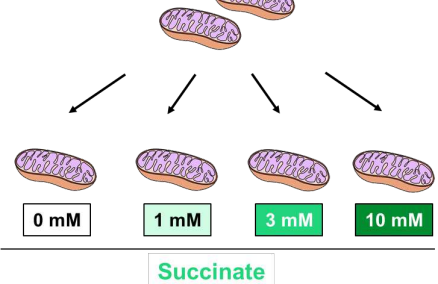

195 b

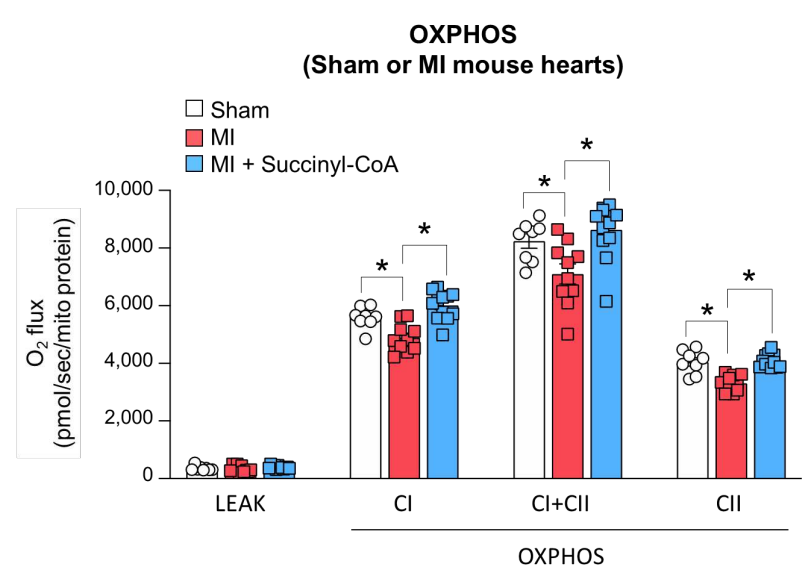

d

OXPHOS

(Non-operated mouse hearts)

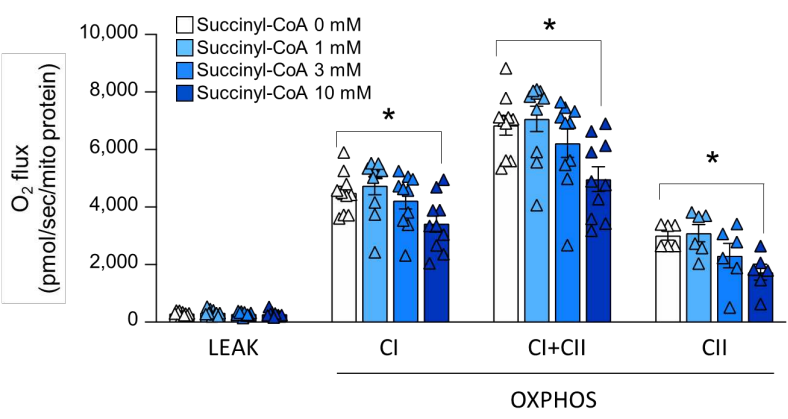

f

OXPHOS

(Non-operated mouse hearts)

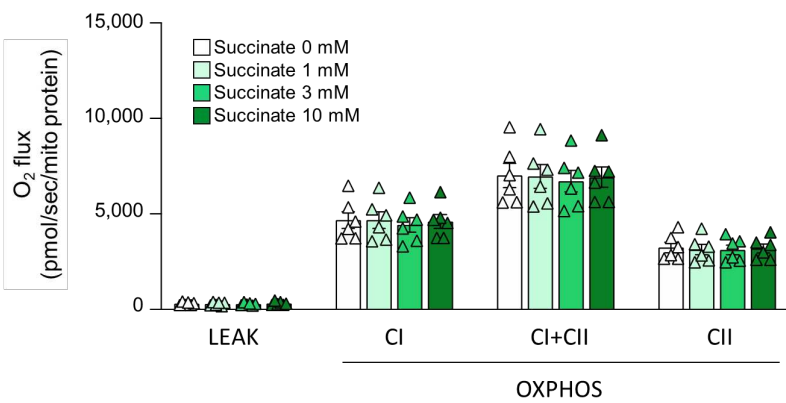


Fig. 3. Reduced succinyl-CoA level impairs OXPHOS activities of myocardial mitochondrial OXPHOS activities of MI mice and sham mice, and their responses to the addition of succinyl-CoA (a); and the actual results (b). c-f, Experimental scheme to measure myocardial mitochondrial OXPHOS activities in non-operated mice in response to the addition of succinyl-CoA (c) and succinate (e); and the actual results $(\mathbf{d}, \mathbf{f})$. In $\mathbf{b}, \mathbf{d}$, and $\mathbf{f}$, each data point in the dot plot represents one individual mouse sample. Data are shown as the mean \pm s.e.m. Data were analyzed by one-way analysis of variance (ANOVA) with the Tukey's test (b), or one-way ANOVA with the Dunnett's test (d). ${ }^{*} P$ $<0.05$. LEAK, leak state

\section{ALA prevents HF progression of MI mice (38/40 characters)}

Based on our results, we next analyzed effective treatments of HF that modify mitochondrial dysfunction (i.e., reduced succinyl-CoA) in MI mice. Succinyl-CoA itself is too unstable to be used as a drug, and the administration of excess succinate has been shown to be harmful to mitochondria and to promote their fragmentation and dysfunction $^{22}$. Based on the mechanism of succinyl-CoA dysregulation in MI mice, we tested the effects of ALA. The administration of ALA to MI mice in their drinking water, which was initiated immediately after coronary artery ligation and was continued for 4 weeks, significantly improved their left ventricular (LV) function and treadmill running capacity (i.e., systemic exercise capacity), and prolonged their survival (Fig. 4a-c). Likewise, the enlargement of LV-end-diastolic diameter (LVEDD), a parameter of LV remodeling, also tended to be prevented in MI mice with ALA treatment (Extended Data 
Fig. 2). Molecularly, both CI- and CII-linked OXPHOS capacities of myocardial

221 mitochondria were significantly improved in MI mice with ALA treatment (Fig. 4d). The

222 administration of ALA also appeared to sufficiently restore succinyl-CoA levels of the

223 myocardial mitochondria of MI mice, although a statistically significant difference was

224 not observed between MI mice with and without ALA treatment, possibly because of the

225 fluctuation in succinyl-CoA levels of MI mice without ALA treatment (Fig. 4e). There

226 was no significant difference in mitochondrial heme levels between MI mice with and

227 without ALA treatment (Fig. 4f). Taken together, the administration of ALA might be an

228 effective treatment for the prevention of HF progression after MI. ALA functions as an

229 intermediate of the heme synthesis from succinyl-CoA, as mentioned earlier. Our results

230 hence also support the notion that the decrease in succinyl-CoA level in MI mice may be

231 caused, at least in part by the excessive consumption of succinyl-CoA outside of the TCA

232 cycle, such as in heme synthesis. 

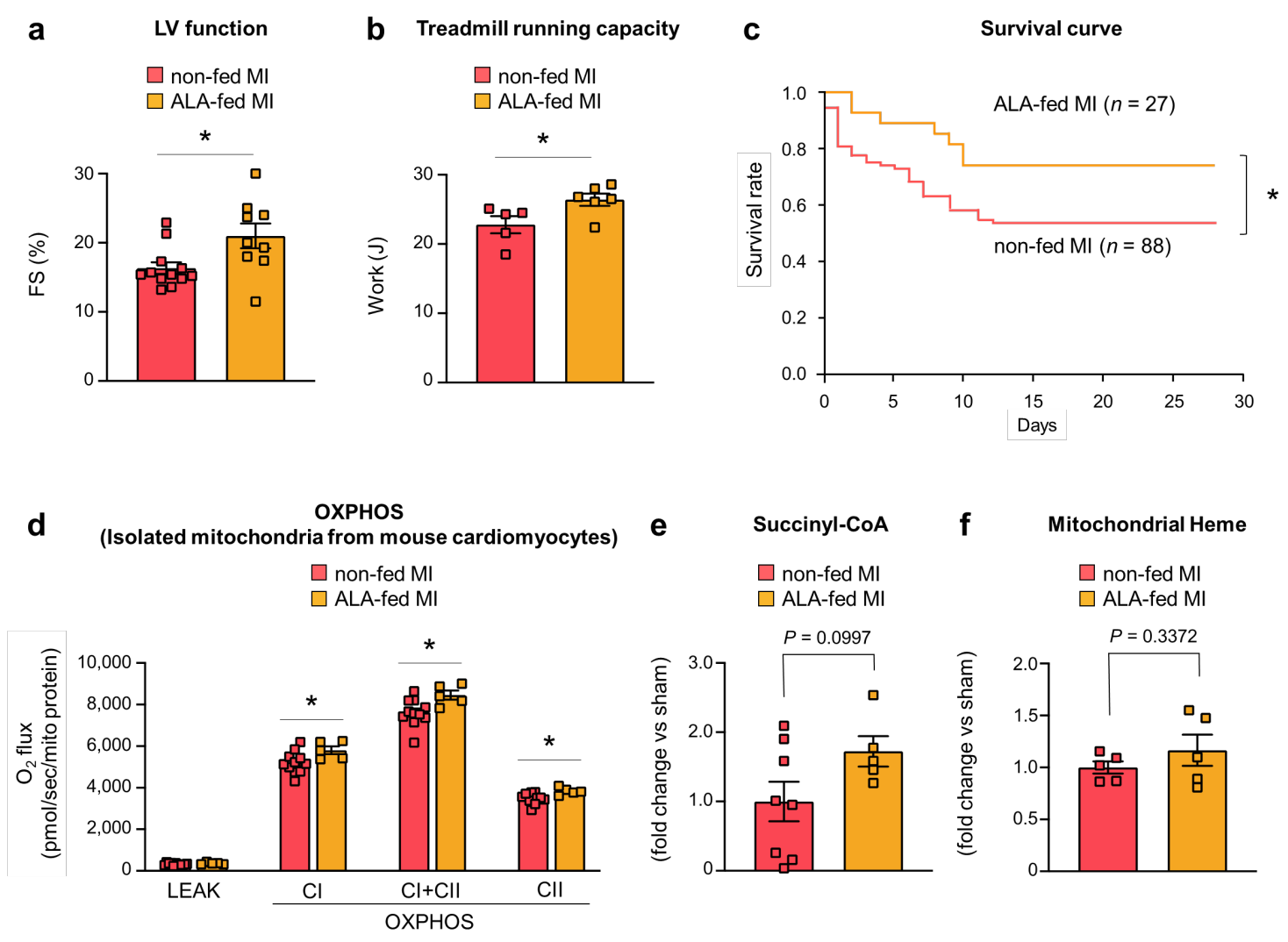

g

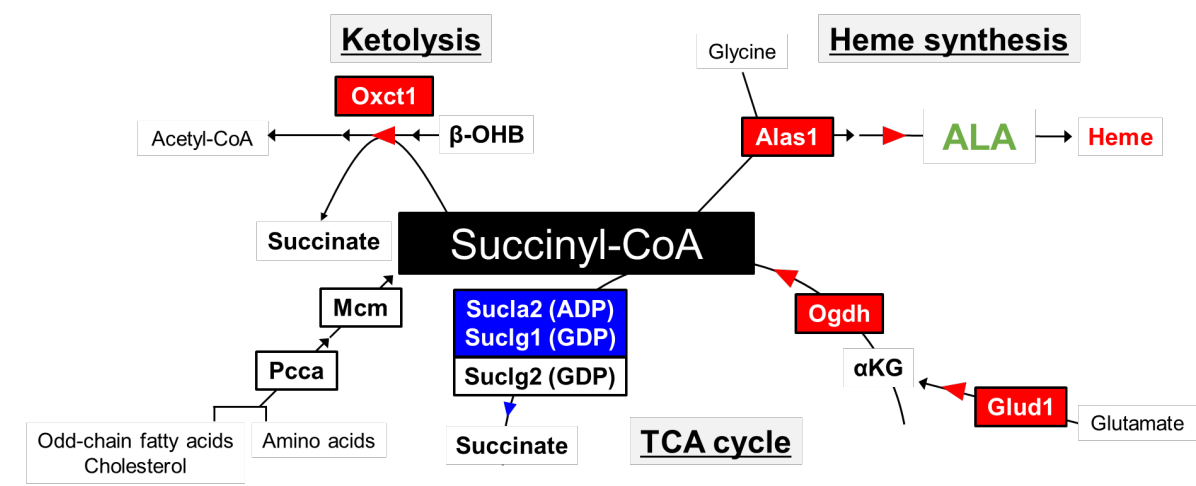

234 Fig. 4. Therapeutic effects of ALA against MI-induced HF. a-c, Effects of ALA on HF

235 symptoms were analyzed by administering MI mice with or without ALA in their drinking

236 water for 4 weeks, starting immediately after the permanent coronary artery ligation; and

237 then measuring their LV function (i.e., the percent fractional shortening (\%FS)) (a),

238 treadmill running capacity (b), and survival (c). d-f, Effects of ALA on OXPHOS activities (d), relative succinyl-CoA levels (e), and relative heme levels (f), measured in 
myocardial mitochondria isolated from ALA-fed or non-fed MI mice. In a, b, d-f, data

241 are shown as the mean \pm s.e.m.. Each data point in the dot plots represents one individual

242 mouse sample. Data were analyzed by the two-tailed Student $t$-test $(\mathbf{a}, \mathbf{b}, \mathbf{d}, \mathbf{e}, \mathbf{f})$ and two-

243 side log-rank test $(\mathbf{c}) .{ }^{*} P<0.05$. g. A proposed model of the metabolic shifts in myocardial

244 mitochondria during chronic HF, whereby we demonstrated that ALA administration to

245 mice can improve succinyl-CoA levels and OXPHOS activities, as well as heart function,

246 and prolong survival.

\section{Extended data Fig. 2}
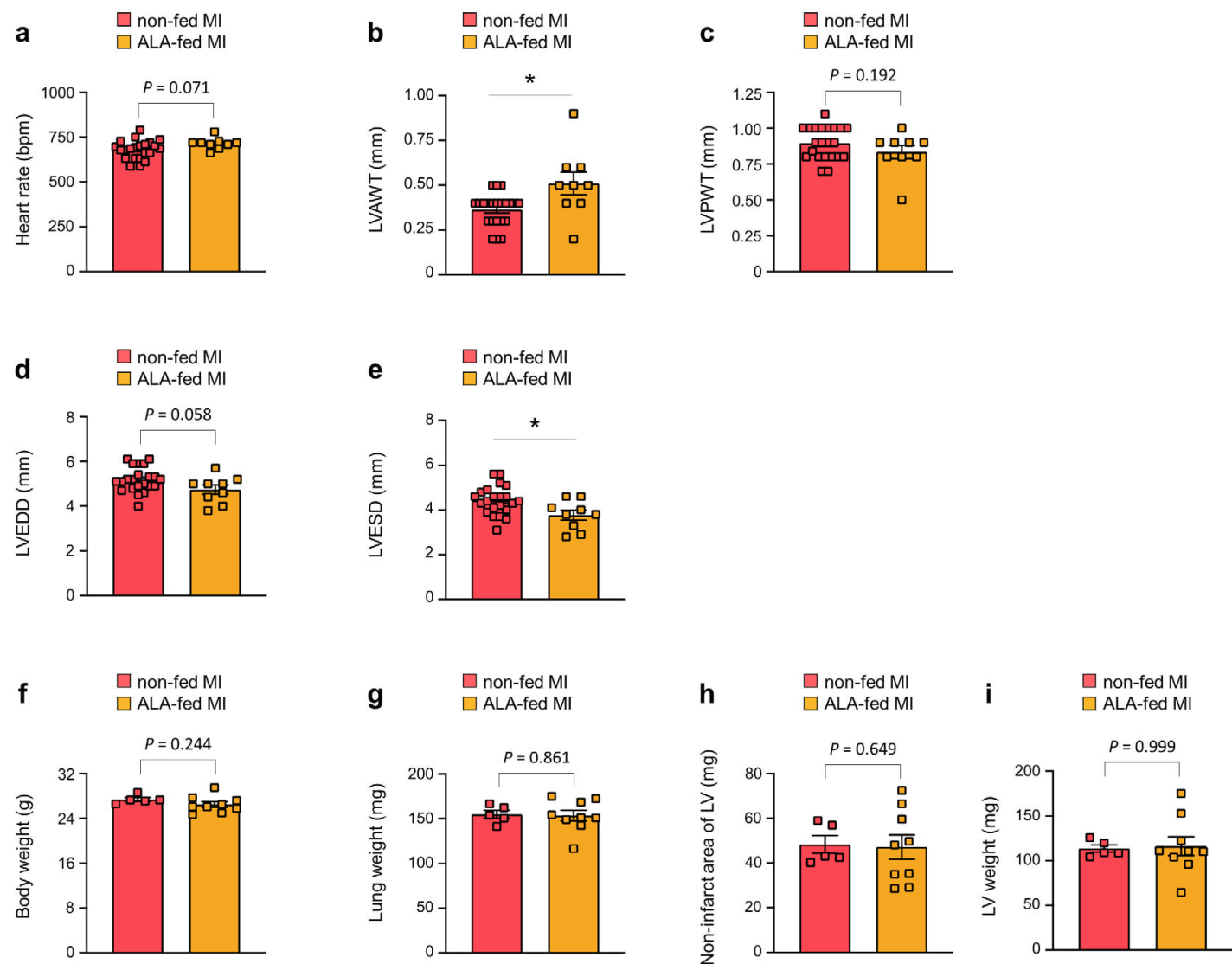

Extended Data Figure 2. Cardiac parameters of ALA-fed and non-fed MI mice. MI 
251 (ALA-fed and non-fed, respectively), starting immediately after the coronary ligation, 252 and then the following cardiac parameters were measured: heart rate (a), LVAWT (b), 253 LVPWT (c), LVEDD (d), LVESD (e), body weight (f), lung weight (g), non-infarct area 254 of LV weight (h), and LV weight (i).

\section{Disturbed protein succinylation in HF (38/40 characters)}

Succinyl-CoA is known as a source of protein succinylation ${ }^{23,24}$. Succinylation occurs predominantly on mitochondrial proteins in the heart, and may affect some of their enzymatic activities ${ }^{25}$. Decreased protein succinylation in the cardiac myofibrils of failing human hearts has been reported previously ${ }^{6}$. Likewise, overall levels of protein succinylation were significantly lower in the myocardial mitochondria of MI mice than in sham mice, and the incubation of isolated mitochondria from MI mice with $1 \mathrm{mM}$ succinyl-CoA significantly increased protein succinylation (Extended Data Fig. 3b). In contrast, the stoichiometry of the succinylation of various proteins is unlikely to be constantly decreased in MI, as reported previously ${ }^{6}$. We observed that the succinylation of many TCA cycle enzymes (Extended Data Fig. 3f) and mitochondrial membrane proteins (Extended Data Fig. 3g) tended to be increased in MI mice. Sirtuin 5 (Sirt5) is the main enzyme that catalyzes Lys desuccinylation in mitochondria ${ }^{26}$. Levels of Sirt5 tended to be downregulated in MI mice, although we did not see a clear difference between MI mice and sham mice (Extended Data Fig. 4). Furthermore, although Sirt5 was reported to suppress SDH activity ${ }^{26}$, we did not observe a decrease in Sdha or Sdhb succinylation in MI mice (Extended Data Fig. 3e). However, we could not rule out the possibility that some artificial events, likely caused by in vitro mitochondrial 
manipulations, were involved in these changes. It may be noteworthy, however, that the

276 incubation of MI mitochondria with succinyl-CoA tended to decrease the succinylation

277 of TCA cycle enzymes and membrane proteins, which appeared to show increased 278 succinylation in MI mice compared with sham mice (Extended Data Fig. 3f, g). On the 279 other hand, it should also be noted that incubation with succinyl-CoA appears to cause 280 further dysregulation of the succinylation stoichiometry of some other proteins, including 281 Sdhb (Extended Data Fig. 3e). Collectively, our results suggest that processes regulating 282 the succinylation/desuccinylation of myocardial mitochondrial proteins might undergo 283 complicated, and perhaps at least partly irreversible changes in MI mice, in which the 284 dysregulation of succinyl-CoA metabolism is likely to be among the causes. Whether the 285 perturbed stoichiometry of mitochondrial protein succinylation affects cardiac functions requires further analyses. 


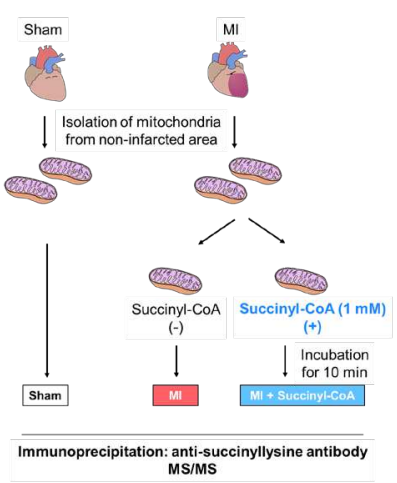

c

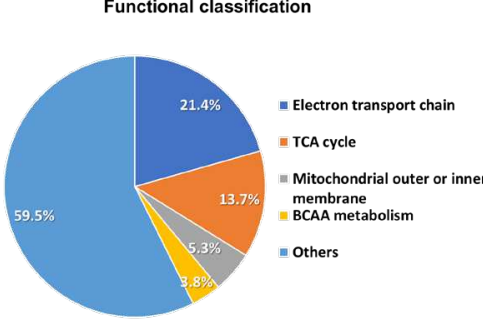

e

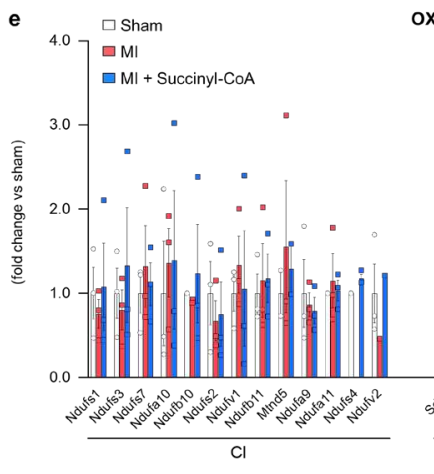

f
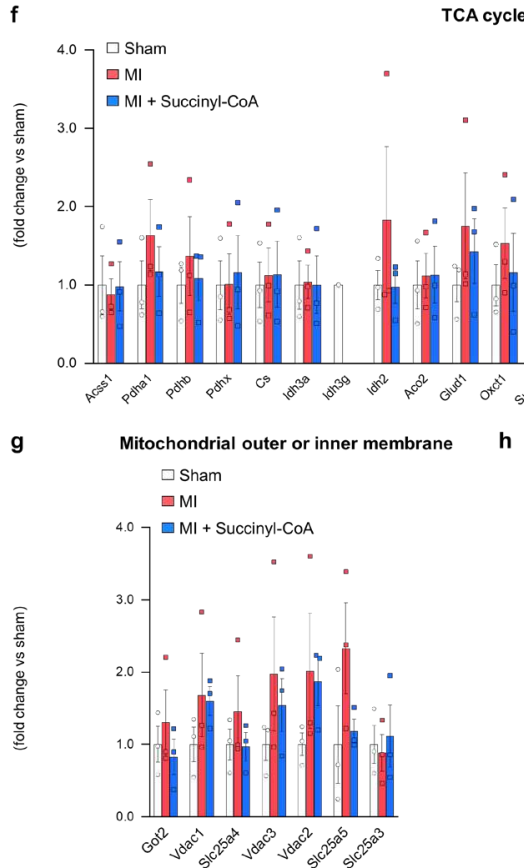

TCA cycle
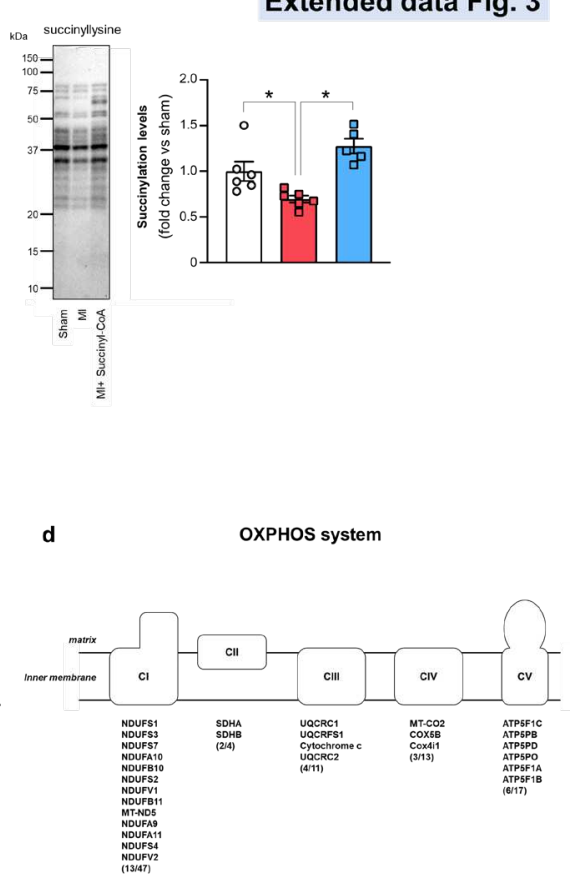

OXPHOS system

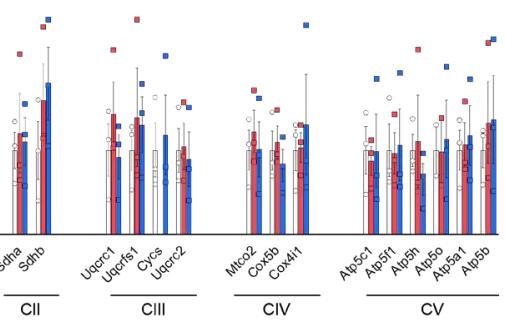


Extended Data Figure 3. Perturbation of protein succinylation in myocardial mitochondria during chronic HF. a, b, Experimental scheme to compare protein

291

292

293

294

295

296

297

298

299

300

301

302

303

304

succinylation in myocardial mitochondria isolated from MI mice with those from sham mice, and their changes in response to succinyl-CoA (a); and the actual results (b). In $\mathbf{b}$, a representative anti-succinyllysine immunoblot of mitochondrial proteins after their separation by SDS-gel electrophoresis is shown on the left, and results of the quantification of all blots is shown on the right ( $n=5$ for each set of samples). c, Percentage of succinylated mitochondrial proteins classified based on their function. d, The name of each protein involved in each component of mitochondrial OXPHOS. e-h, Relative levels of succinylation of each myocardial mitochondria protein in MI mice and sham mice, and changes in the succinylation of proteins of the OXPHOS system (e), the TCA cycle (f), outer and inner membrane components (g), and those involved in branched-chain amino acid (BCAA) metabolism (h) in the myocardial mitochondria of MI mice in response to the addition of succinyl-CoA. In $\mathbf{b}, \mathbf{e - h}$, each data point in the dot plot represents one individual mouse sample. Data are shown as the mean \pm s.e.m. In $\mathbf{b}$, one-way ANOVA followed by the Tukey's test was performed. $* P<0.05$ 


\section{Extended data Fig. 4}
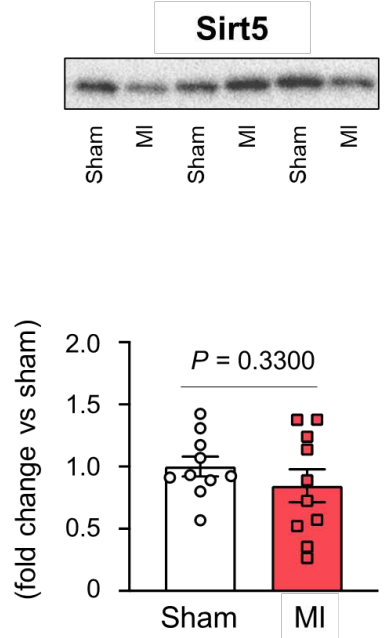

306 Extended Data Figure 4. No significant changes in Sirt5 levels in myocardial

307 mitochondria during chronic HF. A representative result of an immunoblot using an 308 anti-Sirt5 antibody of myocardial mitochondria proteins prepared from MI mice and sham 309 mice (upper panel); and results of the quantification of all blots (lower panel). Each data 310 point in the dot plot represents one individual mouse sample. Data are shown as the mean $311 \pm$ s.e.m. 


\section{Extended data Fig. 5 (internal control)}
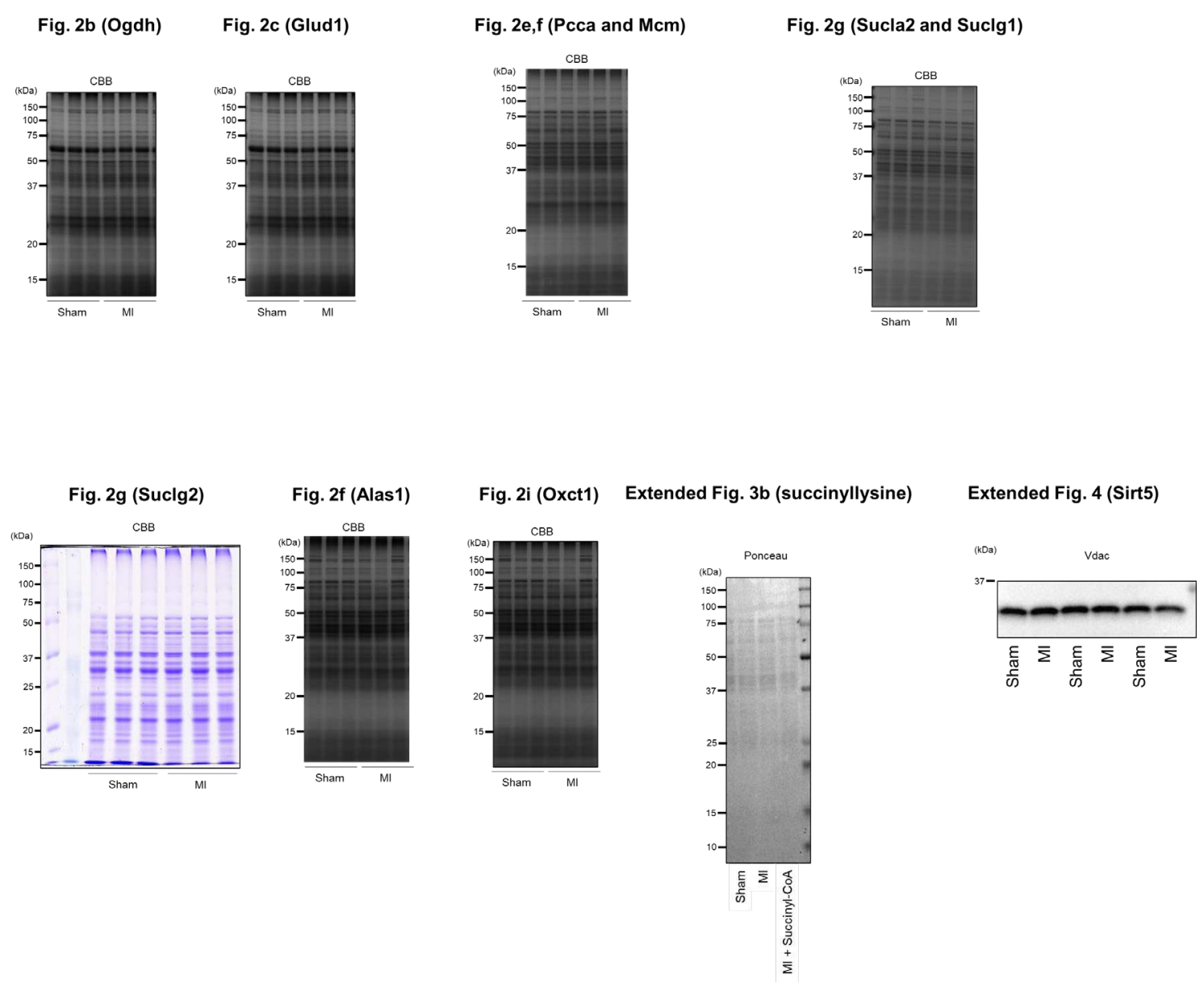

Extended Data Figure 5. Full size scans of the internal controls of the immunoblots

315 shown in the figures of this paper.

\section{Discussion}

319 In this study, we identified previously unknown mechanisms underlying the metabolic 320 dysfunction occurring in myocardial mitochondria, using a well-established mouse model 321 of chronic HF. This dysfunction appeared to be mainly attributable to the altered expression levels of enzymes involved in mitochondrial succinyl-CoA metabolism (Fig. 
3421 Chioncel, O. et al. Clinical phenotypes and outcome of patients hospitalized for

$4 \mathrm{~g}$ ), and a selective decrease in succinyl-CoA level was frequently observed in myocardial mitochondria during chronic HF, thus causing OXPHOS impairment. The metabolic changes that occur in chronic HF, such as ketolysis, also occur in healthy conditions, such as during strenuous exercise, although whether the same enzyme level changes also occur under healthy conditions remains unknown. Intriguingly, we also identified a selective reduction of the ADP-specific isoform of succinyl-CoA synthetase in chronic HF. Although we still do not know the molecular mechanism therein involved, RNASeq data have shown that a particular population of some HF patients demonstrate decreased expression of SUCLG1/SUCLA2, together with increased expression of $A L A S 1$ in their cardiac muscle ${ }^{7,27}$. The dysfunction of succinyl-CoA metabolism in cardiac myofibrils of HF patients was also suggested recently ${ }^{6}$. We demonstrated that nutritional intervention that compensates the altered succinyl-CoA metabolism in chronic HF is a promising method to treat this disease. Therefore, our results, as well as further understanding of the detailed metabolic changes in chronic HF, will facilitate the development of more natural treatments, as well as the prevention of the progression of $\mathrm{HF}^{28}$. Succinyl-CoA is the most abundant acyl-CoA in the heart ${ }^{29}$. Whether the mitochondrial dysfunction occurring in HF affects the histone acylations involved in epigenetic control ${ }^{13}$ should also be clarified in the future $e^{30}$. acute heart failure: the ESC Heart Failure Long-Term Registry. Eur J Heart Fail 19, 1242-1254, doi:10.1002/ejhf.890 (2017).

2 Brown, D. A. et al. Expert consensus document: Mitochondrial function as a therapeutic target in heart failure. Nat Rev Cardiol 14, 238-250, 
doi:10.1038/nrcardio.2016.203 (2017).

3 Okonko, D. O. \& Shah, A. M. Heart failure: mitochondrial dysfunction and oxidative stress in CHF. Nat Rev Cardiol 12, 6-8, doi:10.1038/nrcardio.2014.189 (2015).

4 Bertero, E. \& Maack, C. Metabolic remodelling in heart failure. Nat Rev Cardiol 15, 457-470, doi:10.1038/s41569-018-0044-6 (2018).

5 Stojanovski, B. M. et al. 5-Aminolevulinate synthase catalysis: The catcher in heme biosynthesis. Mol. Genet. Metab. 128, 178-189, doi:10.1016/j.ymgme.2019.06.003 (2019).

6 Ali, H. R. et al. Defining decreased protein succinylation of failing human cardiac myofibrils in ischemic cardiomyopathy. J. Mol. Cell. Cardiol. 138, 304317, doi:10.1016/j.yjmcc.2019.11.159 (2020).

7 Sweet, M. E. et al. Transcriptome analysis of human heart failure reveals dysregulated cell adhesion in dilated cardiomyopathy and activated immune pathways in ischemic heart failure. BMC Genomics 19, 812, doi:10.1186/s12864-018-5213-9 (2018).

8 Chouchani, E. T. et al. Ischaemic accumulation of succinate controls reperfusion injury through mitochondrial ROS. Nature 515, 431-435, doi:10.1038/nature13909 (2014).

9 Martin, J. L. et al. Succinate accumulation drives ischaemia-reperfusion injury during organ transplantation. Nat Metab 1, 966-974, doi:10.1038/s42255-0190115-y (2019).

10 Zhang, J. et al. Accumulation of Succinate in Cardiac Ischemia Primarily Occurs via Canonical Krebs Cycle Activity. Cell Rep 23, 2617-2628, 
doi:10.1016/j.celrep.2018.04.104 (2018).

11 Matsumoto, J. et al. Brain-Derived Neurotrophic Factor Improves Limited Exercise Capacity in Mice With Heart Failure. Circulation 138, 2064-2066, doi:10.1161/CIRCULATIONAHA.118.035212 (2018).

$12 \mathrm{Yu}, \mathrm{X}$. et al. MARK4 controls ischaemic heart failure through microtubule detyrosination. Nature 594, 560-565, doi:10.1038/s41586-021-03573-5 (2021).

13 Trefely, S., Lovell, C. D., Snyder, N. W. \& Wellen, K. E. Compartmentalised acyl-CoA metabolism and roles in chromatin regulation. Mol Metab 38, 100941, doi:10.1016/j.molmet.2020.01.005 (2020).

14 Burch, J. S. et al. Glutamine via alpha-ketoglutarate dehydrogenase provides succinyl-CoA for heme synthesis during erythropoiesis. Blood 132, 987-998, doi:10.1182/blood-2018-01-829036 (2018).

15 Aubert, G. et al. The Failing Heart Relies on Ketone Bodies as a Fuel. Circulation 133, 698-705, doi:10.1161/CIRCULATIONAHA.115.017355 (2016).

16 Horton, J. L. et al. The failing heart utilizes 3-hydroxybutyrate as a metabolic stress defense. JCI Insight 4, doi:10.1172/jci.insight.124079 (2019).

17 Xie, J., Liu, X., Li, Y., Liu, Y. \& Su, G. Validation of RT-qPCR reference genes and determination of Robo4 expression levels in human retinal endothelial cells under hypoxia and/or hyperglycemia. Gene 585, 135-142, doi:10.1016/j.gene.2016.03.047 (2016).

18 Khechaduri, A., Bayeva, M., Chang, H. C. \& Ardehali, H. Heme levels are increased in human failing hearts. J. Am. Coll. Cardiol. 61, 1884-1893, doi:10.1016/j.jacc.2013.02.012 (2013). 
19 Evans, M., Cogan, K. E. \& Egan, B. Metabolism of ketone bodies during exercise and training: physiological basis for exogenous supplementation. $J$ Physiol 595, 2857-2871, doi:10.1113/JP273185 (2017).

20 Maekawa, S. et al. Linoleic acid improves assembly of the CII subunit and CIII2/CIV complex of the mitochondrial oxidative phosphorylation system in heart failure. Cell Commun Signal 17, 128, doi:10.1186/s12964-019-0445-0 (2019).

21 Martinez-Reyes, I. \& Chandel, N. S. Mitochondrial TCA cycle metabolites control physiology and disease. Nat Commun 11, 102, doi:10.1038/s41467-019$13668-3(2020)$

22 Pfleger, J., He, M. \& Abdellatif, M. Mitochondrial complex II is a source of the reserve respiratory capacity that is regulated by metabolic sensors and promotes cell survival. Cell Death Dis 6, e1835, doi:10.1038/cddis.2015.202 (2015).

23 Wagner, G. R. \& Payne, R. M. Widespread and enzyme-independent Nepsilonacetylation and Nepsilon-succinylation of proteins in the chemical conditions of the mitochondrial matrix. J. Biol. Chem. 288, 29036-29045, doi:10.1074/jbc.M113.486753 (2013).

24 Yang, Y. \& Gibson, G. E. Succinylation Links Metabolism to Protein Functions. Neurochem. Res. 44, 2346-2359, doi:10.1007/s11064-019-02780-х (2019).

25 Wagner, G. R. et al. A Class of Reactive Acyl-CoA Species Reveals the Nonenzymatic Origins of Protein Acylation. Cell Metab 25, 823-837 e828, doi:10.1016/j.cmet.2017.03.006 (2017).

26 Park, J. et al. SIRT5-mediated lysine desuccinylation impacts diverse metabolic pathways. Mol. Cell 50, 919-930, doi:10.1016/j.molcel.2013.06.001 (2013). 
41927 Ramirez Flores, R. O. et al. Consensus Transcriptional Landscape of Human

$420 \quad$ End-Stage Heart Failure. J Am Heart Assoc 10, e019667, $421 \quad$ doi:10.1161/JAHA.120.019667 (2021).

42228 Takada, S., Sabe, H. \& Kinugawa, S. Abnormalities of Skeletal Muscle,

423 Adipocyte Tissue, and Lipid Metabolism in Heart Failure: Practical Therapeutic 424 Targets. Front Cardiovasc Med 7, 79, doi:10.3389/fcvm.2020.00079 (2020).

42529 Sadhukhan, S. et al. Metabolomics-assisted proteomics identifies succinylation and SIRT5 as important regulators of cardiac function. Proc. Natl. Acad. Sci. U. S. A. 113, 4320-4325, doi:10.1073/pnas.1519858113 (2016).

$42830 \quad$ Gilsbach, R. et al. Distinct epigenetic programs regulate cardiac myocyte development and disease in the human heart in vivo. Nat Commun 9, 391, doi:10.1038/s41467-017-02762-z (2018). 
431

432

433

434

435

436

437

438

439

440

441

442

443

444

445

446

447

448

449

450

451

452

453

454

\section{Methods}

\section{Animal procedures and ethics statement}

Animal experiments were performed according to a protocol approved by the Animal Care Committee of Hokkaido University (study approval no.: 16-0115). C57BL/6J mice were used in all experiments, and were housed under standard conditions (temperature: $23-25^{\circ} \mathrm{C}$, and humidity: $40 \%-60 \%$ ) on a 12 -h light/dark cycle.

\section{In vivo HF mouse model}

As an in vivo HF model, a coronary ligated model was used, as described previously ${ }^{11}$. Permanent left anterior descending (LAD) coronary artery ligation was performed on the mice. Male C57BL/6J mice (9-12 weeks old; CLEA Japan) were anesthetized with an intraperitoneal injection of a mixture of $0.3 \mathrm{mg} / \mathrm{kg}$ body weight of medetomidine (Kyoritsu, Dorbene $^{\circledR}$ ), $4.0 \mathrm{mg} / \mathrm{kg}$ body weight of midazolam (Astellas, Dormicum ${ }^{\circledR}$ ), and $5.0 \mathrm{mg} / \mathrm{kg}$ body weight of butorphanol (Meiji, Vetorphale ${ }^{\circledR}$ ) (MMB mixture), and then intubated and ventilated with air (supplemented with oxygen) using a small-animal respirator. The adequacy of anesthesia was monitored by the pedal withdrawal reflex. A thoracotomy was performed in the fourth left intercostal space. Then, the left ventricle was visualized and the pericardial sac was ruptured to expose the LAD coronary artery. The LAD was permanently ligated using a 4-0 Prolene suture (Covidien, Sofsilk ${ }^{\mathrm{TM}}$ VS709). The suture was passed approximately $0.5 \mathrm{~mm}$ below the tip of the left auricle. Shamoperated mice, which were used as controls, underwent thoracotomy of the heart as in coronary-ligated mice, except that their arteries were not tied. The thoracotomy was closed with 8-0 Prolene sutures (Akiyama Medical MFG, M6-80B2). The endotracheal tube was removed once spontaneous respiration resumed, and the mice were placed in a 
warm recovery cage maintained at $37^{\circ} \mathrm{C}$ until they were completely awake. After 4 weeks, mice were subjected to analyses. For biochemical analysis, mice were sacrificed with an intraperitoneal injection of the MMB mixture, and the heart was then excised and subjected to analyses. Regarding the LVs of HF mice, the infarct areas were excluded from the biochemical analyses, and only noninfarct areas were used for the experiments. Blood samples of the mice were collected from the inferior vena cava before euthanization by deep anesthesia with the MMB mixture, as described previously ${ }^{31}$.

\section{Metabolite extraction from mouse heart}

Mice were sacrificed by cervical dislocation under general anesthesia. Mouse LVs, excluding the infarcted areas, were isolated within 2 min after cervical dislocation, and rapidly cryopreserved with liquid nitrogen. Pieces of the mouse LV (40-50 mg) were snap-frozen in liquid nitrogen and crushed using a MultiBeads Shocker (Bio Medical Science) at 2,000 rpm for $10 \mathrm{sec}$. The crushed powder was then dissolved in $1 \mathrm{~mL}$ of icecold $80 \%$ methanol per $100 \mathrm{mg}$ tissue weight, sonicated five times (five rounds of sonication for 30 secs and cooling for 30 secs) using a BIORUPTOR (Cosmo Bio), and centrifuged at $21,500 \mathrm{~g}$ for $5 \mathrm{~min}$ at $4{ }^{\circ} \mathrm{C}$. The supernatants were then collected and subjected to LC-MS analyses.

\section{Metabolite analysis by LC-MS}

To measure the TCA cycle intermediates, heart lysate supernatants (equivalent to approximately $1.5 \mathrm{mg}$ of the heart) were applied and separated on an ACQUITY BEH C18 column $\left(100 \mathrm{~mm} \times 2.1 \mathrm{~mm}, 1.7 \mu \mathrm{m}\right.$, Waters $\left.{ }^{\mathrm{TM}}, 186002352\right)$ and analyzed using an LCMS 8040 instrument (Shimadzu) by multiple reaction monitoring of 98 specific 
negative ions, as described previously ${ }^{32}$. The mobile phase consisted of $15 \mathrm{mM}$ acetic acid and $10 \mathrm{mM}$ tributylamine in 3\% methanol solution (A) and $100 \%$ methanol (B). The gradient elution program was as follows: $0-6 \mathrm{~min}, 0 \% \mathrm{~B}$; 6-26 $\mathrm{min}, 0 \%-90 \% \mathrm{~B}$; decreased to $0 \% \mathrm{~B}$ and maintained until $15 \mathrm{~min}$. The flow rate was $0.3 \mathrm{~mL} / \mathrm{min}$, and the column oven temperature was $40^{\circ} \mathrm{C}$. Parameters for the negative electrospray ionization mode were as follows: drying gas flow rate, $15 \mathrm{~L} / \mathrm{min}$; nebulizer gas flow rate, $3 \mathrm{~L} / \mathrm{min}$; heating gas flow rate, $10 \mathrm{~L} / \mathrm{min}$; desolvation line temperature, $250{ }^{\circ} \mathrm{C}$; and heat block temperature, $400{ }^{\circ} \mathrm{C}$; collision induced dissociation gas, $230 \mathrm{kPa}$. Data processing was performed using LabSolutions software (Shimadzu) and signal intensities were standardized against the signals of MES.

\section{Isolation of mitochondria from mouse heart}

Mitochondria were isolated as described previously ${ }^{20}$. Briefly, excised mouse hearts were quickly minced using scissors for $4 \mathrm{~min}$, and incubated with $0.1 \mathrm{mg} / \mathrm{mL}$ proteinase (Sigma-Aldrich, $\mathrm{P} 8038$ ) for $2 \mathrm{~min}$ at $4{ }^{\circ} \mathrm{C}$ in mitochondrial isolation buffer containing 50 mM Tris- $\mathrm{HCl}\left(\mathrm{pH}\right.$ 7.4), $100 \mathrm{mM} \mathrm{KCl,} 100 \mathrm{mM}$ sucrose, 1 mM KH $\mathrm{PO}_{4}, 0.1 \mathrm{mM}$ EGTA, and $0.2 \%$ bovine serum albumin. They were then gently homogenized using a motordriven Teflon pestle in a glass chamber (Wheaton, 358039) with six strokes, and centrifuged at $700 \mathrm{~g}$ for $10 \mathrm{~min}$. Supernatants were recentrifuged at $10,000 \mathrm{~g}$ for $10 \mathrm{~min}$, and pellets were then suspended in the mitochondrial isolation buffer, after washing briefly once with the same buffer, and centrifuged again at 7,000 $\mathrm{g}$ for $3 \mathrm{~min}$. The resulting pellets were resuspended in a buffer containing $10 \mathrm{mM}$ Tris- $\mathrm{HCl}(\mathrm{pH} 7.4), 225 \mathrm{mM}$ mannitol, $75 \mathrm{mM}$ sucrose, and 0.1 mM EDTA, and subjected to analyses. All procedures, except for the preincubation with proteinase, were performed at $4{ }^{\circ} \mathrm{C}$. Protein 
concentrations of the samples were measured using the bicinchoninic acid assay (Thermo Fisher Scientific, 23225).

\section{Immunoblotting analysis}

Immunoblotting analysis was performed as described previously ${ }^{33}$. Briefly, mitochondrial proteins were denatured in Laemmli buffer (100 mM Tris- $\mathrm{HCl}$ [pH 6.8], 4\% SDS, 4\% glycerol, $0.05 \%$ bromophenol blue, $12 \%$ 2-mercaptoethanol) at $100{ }^{\circ} \mathrm{C}$ for $5 \mathrm{~min}$, separated by SDS-PAGE using Any $\mathrm{kD}^{\mathrm{TM}}$ Criterion $^{\mathrm{TM}}$ precast gels (Bio-Rad, 5671122J10), and transferred to polyvinylidene fluoride membranes (Bio-Rad, cat. no.: 1704156). Membranes were then blocked for $1 \mathrm{~h}$ at room temperature in TBS-T buffer $(0.1 \%$ Tween-20 in $1 \times$ PBS $)$ containing $3 \%$ milk, and incubated with primary antibodies diluted in TBS-T with $3 \%$ milk, overnight at $4{ }^{\circ} \mathrm{C}$. Primary antibodies used were as follows: Sucla2 (Abcam, ab202582, 1:1,000 dilution), Suclg1 (Abcam, ab204432, 1:1,000 dilution), Suclg2 (Abcam, ab241375, 1:1,000 dilution), Glud1 (Abcam, ab 168352, 1:1,000 dilution), Ogdh (Abcam, ab137773, 1:1,000 dilution), Alas1 (Abcam, ab84962, 1:1,000 dilution), Oxct-1 (Abcam, ab105320, 1:1,000 dilution), Pcca (Abcam, ab187686, 1:1,000 dilution), Mcm (Abcam, ab134956, 1:1,000 dilution), Sirt5 (Abcam, ab13697, 1:1,000 dilution) and succinyllysine (PTM BIO, PTM419, 1:1,000 dilution). After three washes with TBS-T, membranes were then incubated with a horseradish peroxide-conjugated secondary antibody (Abcam, ab97051, 1:20,000 dilution, or Santa Cruz, sc-2314, 1:5,000 dilution) in TBS-T with $3 \%$ milk, for $1 \mathrm{~h}$ at room temperature. After washing, peroxide-conjugated Abs retained on membranes were visualized with the enhanced chemiluminescence kit (Thermo Fisher Scientific, 32106 or 34075) coupled with ChemiDoc XRS ${ }^{+}$(Bio-Rad). Densities of the signals were quantified with Image $\mathrm{J}$ 
software (U.S. National Institutes of Health). Expression levels of the proteins are shown as values normalized by the protein level of voltage-dependent anion channel, which is a representative mitochondrial marker (Cell Signaling Technology, 4866, 1:1,000 dilution), or total amounts of proteins measured by Coomassie brilliant blue (CBB) staining (BioRad, 1610786) or Ponceau-S staining (Beacle, BCL-PSS-01).

\section{Measurement of mitochondrial OXPHOS activities}

To assess mitochondrial OXPHOS, respiration capacities of isolated mitochondria were measured with a high-resolution respirometer (Oxygraph-2k, Oroboros) ${ }^{11}$. Isolated mitochondria (approximately $30-100 \mu \mathrm{g}$ ) were placed into the respirometer chamber, filled with $2 \mathrm{~mL}$ of MiR05 medium (110 mmol/L sucrose, $60 \mathrm{mmol} / \mathrm{L}$ K-lactobionate, 0.5 mmol/L EGTA, 0.1\% BSA, $3 \mathrm{mmol} / \mathrm{L} \mathrm{MgCl}_{2}, 20 \mathrm{mmol} / \mathrm{L}$ taurine, $10 \mathrm{mmol} / \mathrm{L} \mathrm{KH}_{2} \mathrm{PO}_{4}$, $20 \mathrm{mmol} / \mathrm{L}$ HEPES, pH 7.1), and then incubated with chemicals at $37^{\circ} \mathrm{C}$ in the following order (final concentrations are indicated): (1) $2 \mathrm{mM}$ malate, $5 \mathrm{mM}$ pyruvate, $10 \mathrm{mM}$ glutamate (complex I-linked substrates), (2) $10 \mathrm{mM}$ ADP, and (3) $10 \mathrm{mM}$ succinate (complex II-linked substrate) and $0.5 \mu \mathrm{M}$ rotenone (a complex I inhibitor). The $\mathrm{O}_{2}$ consumption rates (i.e., respiration rates) were expressed as $\mathrm{O}_{2}$ flux normalized to the mitochondrial protein concentration $(\mu \mathrm{g} / \mu \mathrm{L})$. DatLab software (Oroboros) was used for data acquisition and analysis.

\section{Measurement of mitochondrial heme}

Intra-mitochondrial heme was quantified using the QuantiChrom ${ }^{\mathrm{TM}} \mathrm{Heme}$ Assay Kit (BioaAssay Systems, DIHM-250), according to the manufacture's instruction. Briefly, isolated mitochondria were mixed with $200 \mu \mathrm{L}$ of reaction mixture provided with the 

assay kit, and incubated for $5 \mathrm{~min}$ at room temperature. Optical densities at $400 \mathrm{~nm}$ were then measured in a microtiter plate reader (Multiskan GO, Thermo Fisher), in which the standard curve was prepared using a heme standard provided by the assay kit.

\section{Measurement of plasma and heart beta-hydroxybutyrate}

$\beta$-OHB levels in the plasma and hearts were measured using the $\beta$-OHB Assay Kit (Abcam, ab180876), according to the manufacture's instruction.

\section{Feeding mice with ALA}

Mice were divided into two groups after coronary ligation; one group was given normalwater, and the other was given water containing $50 \mathrm{mg} / \mathrm{L}$ ALA (Sigma Aldrich, A3785), and the amount of ALA ingested was 6 to $8 \mathrm{mg} / \mathrm{kg}$ body weight per day. After 4 weeks, mice were subjected to echocardiography and treadmill running, and then sacrificed with an intraperitoneal injection of the MMB mixture, for subsequent biochemical analyses.

\section{Echocardiography}

Echocardiographic measurements were performed in mice in the conscious state to avoid any effects of anesthesia on cardiac function, as described previously ${ }^{33}$. Briefly, a commercially available echocardiography system (Toshiba Medical Systems, Aplio $^{\mathrm{TM}} 300$ ) was used with a dynamically focused $12-\mathrm{MHz}$ linear array transducer and a depth setting of $2.0 \mathrm{~cm}$. The fur on the chest was removed using depilatory cream and a layer of acoustic coupling gel was applied to the thorax. A two-dimensional parasternal short-axis view was obtained at the levels of the papillary muscles. In general, the clearest views were obtained with the transducer lightly applied to the mid-upper left anterior 
chest wall. The transducer was then gently moved in the cephalad or caudad direction, and angulated until desirable images were obtained. After confirmation that the imaging was on the axis, two-dimensional targeted M-mode tracings were recorded at a paper speed of $50 \mathrm{~mm} / \mathrm{sec}$. The following indexes were analyzed using the software in the echo instrument: LVEDD, LV end-systolic diameter (LVESD), percent fractional shortening $(\% \mathrm{FS})$, heart rate (HR), anterior wall thickness (AWT), and posterior wall thickness (PWT) of the LV.

\section{Treadmill running}

Mice were subjected to treadmill running to assess their whole-body exercise capacity, as previously described ${ }^{31}$. Briefly, each mouse was made to run on a motor-driven treadmill enclosed within a chamber with constant air flow, in which the $\mathrm{O}_{2}$ and $\mathrm{CO}_{2}$ fractions were monitored (Oxymax 2; Columbus Instruments). After a 10-min warm-up at $6 \mathrm{~m} / \mathrm{min}$ at $0^{\circ}$ inclination, the treadmill angle was fixed at $10^{\circ}$ and the speed was gradually increased by $2 \mathrm{~m} / \mathrm{min}$ until the mouse attained exhaustion. Exhaustion was defined as spending more than $10 \mathrm{sec}$ on the electrical shocker plate without attempting to go back onto the treadmill. Work as whole-body exercise capacity was defined as the product of the vertical running distance to exhaustion, and body weight.

\section{Protein succinylation of mitochondria}

Mitochondria isolated from mouse cardiac muscles were denatured in buffer (1\% SDS and $0.07 \%$ 2-mercaptoethanol in PBS) and boiled for $10 \mathrm{~min}$. Mitochondrial proteins were then incubated with an anti-succinyllysine antibody conjugated with agarose beads (PTM Biolabs, PTM419) at $4{ }^{\circ} \mathrm{C}$ with gentle rotation overnight. After washing three times 
599

600

601

602

603

604

605

606

607

608

609

610

611

612

613

614

615

616

617

618

619

620

621

622

with buffer (0.05\% Lauryl Maltoside in PBS), the beads were dissolved in Laemmli buffer and boiled for $5 \mathrm{~min}$. Supernatants were then collected after brief centrifugation, and subjected to MS/MS. After reduction with $10 \mathrm{mM}$ TCEP (FUJIFILM Wako Chemicals, 203-20153) at $100{ }^{\circ} \mathrm{C}$ for $10 \mathrm{~min}$ and alkylation with $50 \mathrm{mM}$ iodoacetamide (FUJIFILM Wako Chemicals, 093-02152) at ambient temperature for $45 \mathrm{~min}$, protein samples were subjected to SDS-PAGE. Electrophoresis was stopped at the migration distance of $2 \mathrm{~mm}$ from the top edge of the separation gel. After CBB-staining, protein bands were excised, destained, and cut finely prior to in-gel digestion with Trypsin/Lys-C Mix (Promega, V5072) at $37{ }^{\circ} \mathrm{C}$ for $12 \mathrm{~h}$. The resulting peptides were extracted from gel fragments and analyzed with Orbitrap Fusion Lumos mass spectrometer (Thermo Scientific) combined with UltiMate 3000 RSLC nano-flow HPLC (Thermo Scientific). Peptides were enriched using $\mu$-Precolumn $(0.3 \mathrm{~mm}$ i.d. $\times 5 \mathrm{~mm}, 5 \mu \mathrm{m}$, Thermo Scientific, 160454) and separated on an AURORA column $(0.075 \mathrm{~mm}$ i.d. $\times 250 \mathrm{~mm}, 1.6 \mu \mathrm{m}$, Ion Opticks Pty, AUR25075C18AC) using the following two-step gradient: $2 \%$ to $40 \%$ acetonitrile for $110 \mathrm{~min}$, followed by $40 \%$ to $95 \%$ acetonitrile for $5 \mathrm{~min}$ in the presence of $0.1 \%$ formic acid. The analytical parameters of Orbitrap Fusion Lumos were set as follows: resolution of full scans $=50,000$, scan range $(\mathrm{m} / \mathrm{z})=350-1,500$, maximum injection time of full scans $=50 \mathrm{msec}$, AGC target of full scans $=4 \times 10^{5}$, dynamic exclusion duration $=30$ sec, cycle time of data dependent MS/MS acquisition $=2 \mathrm{sec}$, activation type $=\mathrm{HCD}$, detector of $\mathrm{MS} / \mathrm{MS}=$ ion trap, maximum injection time of $\mathrm{MS} / \mathrm{MS}=35 \mathrm{msec}$, AGC target of $\mathrm{MS} / \mathrm{MS}=1 \times 10^{4}$

The MS/MS spectra were searched against the Mus musculus protein sequence database in SwissProt using Proteome Discoverer 2.4 software (Thermo Scientific), in which peptide identification filters were set at "false discovery rate $<1 \%$ ". Label-free 
623

624

625

626

627

628

629

630

631

632

633

634

635

636

637

638

639

640

641

642

643

644

645

646

relative quantification analysis of proteins was performed with the default parameters of Minora Feature Detector node, Feature Mapper node, and Precursor Ions Quantifier node in Proteome Discoverer 2.4 software.

\section{Statistics and reproducibility}

Data are expressed as the mean \pm the s.e.m. Statistical analyses were performed using the Student $t$-test for comparisons between two groups, and by ANOVA followed by the Tukey test for comparisons between three groups using GraphPad Prism 6 software (GraphPad, San Diego, CA). The Dunnett's method was used for multiple comparisons with a control group. A univariate linear regression model was used to determine the correlations between two variables. For all animal experiments, all stated replicates are biological replicates. A $p$-value of less than 0.05 was considered to indicate a statistically significant difference between groups. Kaplan-Meier analysis with the log-rank test was performed to compare survival rates among 2 groups for 28 days MI-postsurgery. For ALA treatment studies, mice were randomly assigned to treatment groups. For mass spectrometry analyses, samples were processed in random order and experimenters were blinded to the experimental conditions. All experiments were successfully repeated with similar results at least two or three times.

\section{Data reporting}

No statistical methods were used to predetermine sample size. For in vivo experiments in which grouping was based on surgery, mice in the MI group were randomly chosen for the experiments. For in vivo experiments in which grouping was based on ALA treatment, mice in the MI group were randomly allocated to treatment groups. The investigators 
647

648

649

650

651

652

653

654

655

656

657

658

659

660

661

662

663

664

665

666

667

668

669

670

were not blinded to allocation during the experiments and outcome assessment. Further information on randomization and blinding is available in the Nature Research Reporting Summary linked to this article.

\section{Reporting summary}

Further information on the research design is available in the Nature Research Reporting Summary linked to this article.

\section{Data availability}

All of the associated raw data presented in this paper are available from the corresponding author upon request. Source data are provided with this paper. Full scans for all western blots are provided in Supplementary Information. All other data are available from the corresponding author on reasonable request. Source data are provided with this paper.

31 Nambu, H. et al. Inhibition of xanthine oxidase in the acute phase of myocardial infarction prevents skeletal muscle abnormalities and exercise intolerance.

Cardiovasc. Res. 117, 805-819, doi:10.1093/cvr/cvaa127 (2021).

32 Saito, T. et al. Cardiomyocyte-specific loss of mitochondrial p32/C1qbp causes cardiomyopathy and activates stress responses. Cardiovasc. Res. 113, 11731185, doi:10.1093/cvr/cvx095 (2017).

33 Furihata, T. et al. Cardiac-specific loss of mitoNEET expression is linked with age-related heart failure. Commun Biol 4, 138, doi:10.1038/s42003-021-01675-4 (2021). 
672 Acknowledgments The authors thank Yuki Kimura and Miwako Yamane for their 673 technical assistance, Ayae Oda, Misaki Kihara, Naoko Toshiro, Tsukusu Yamanaka, and 674 Misato Kobayashi for secretarial support, and H.A. Popiel for her critical reading of the 675 manuscript. This work was supported in part by Japanese Grants-In-Aid for Scientific 676 Research (JP17H04758 [S.T.], $18 \mathrm{H} 03187$ [S.K.]), Grant-In-Aid for Challenging 677 Exploratory Research (19K22791 to S.T.), grants from the Japan Foundation for Applied 678 Enzymology (S.T.), the MSD Life Science Foundation (S.T.), the Uehara Memorial 679 Foundation (S.T.), the Cardiovascular Research Fund of Tokyo (S.T.), the Fukuda 680 Memorial Foundation for Medical Research (S.T.), the SENSHIN Medical Research 681 Foundation (S.T.), and JST COI Grant Number JPMJCE1301.

682

683 Author contributions S.T., S.M., S.K., and H.S. conceived the project. S.T., S.M., S.K., 684 and H.S. designed the experiments. S.T., S.M., T.F., N.K., D.S., K.U., H.N., H.Hagiwara., 685 H.Handa., Y.F., S.H., A.F., T.Y., and D.K. collected, assembled, analyzed, and interpreted 686 the data. S.T., S.M., S.K., and H.S. wrote the paper. S.T., S.K., and H.S. critically revised 687 the manuscript. All authors approved the final version of the manuscript and agree to be 688 accountable for all aspects of the work.

689

690 Competing interests The authors declare no competing interests associated with this 691 study. 


\section{Supplementary Files}

This is a list of supplementary files associated with this preprint. Click to download.

- ExtendedDataFiguresSucCoA.pdf

- SupplementaryinformationsuccinylCoA.pdf 